\title{
Insights Into Ototoxicity
}

\section{Analogies to Nephrotoxicity}

\author{
H. DAVID HUMES
}

Departments of Internal Medicine, VA Medical Center and University of Michigan, Ann Arbor, Michigan 48109, USA

\begin{abstract}
The most common ototoxic compounds in clinical practice are aminoglycoside antibiotics, cisplatin, and loop diuretics (ethacrynic acid and furosemide). These agents also have substantial renal effects in the form of nephrotoxicity or diuresis. The understanding of these renal effects may provide insight into ototoxic mechanisms. For aminoglycosides, the renal proximal tubule cell is susceptible due to high concentration achieved and slow clearance with direct effects on phosphoinositide binding and mitochondrial bioenergetics. Pathogenesis appears to involve iron-induced free-radical formation, since iron chelators prevent nephrotoxicity. Analogous effects of aminoglycosides on the inner and outer hair cells have been observed. Cisplatin is also highly concentrated in the proximal tubule cell. Less is known about the direct toxic effects of this agent on renal cells. Insights into mechanisms on renal tubule cells could be directly relevant to the inner ear. The loop diuretics are direct inhibitors of the $\mathrm{Na}^{+}-\mathrm{K}^{+}-2 \mathrm{Cl}^{-}$cotransport system, which also exists in the marginal and dark cells of the stria vascularis, which are responsible for endolymph secretion. The ototoxicity of these agents may be indirect, due to changes in ionic composition and fluid volume within the endolymph.
\end{abstract}

\section{INTRODUCTION}

A striking association exists between the inner ear and the kidney with regards to various disease processes. Three of the most prevalent classes of ototoxic agents, which include aminoglycoside antibiotics, platinum-containing chemotherapeutic agents, and loop diuretics, also have profound toxic or functional effects on the kidney. In addition, a common genetic disease, Alport's syndrome, produces hearing loss and renal disease. Because of this association, insights into the mechanisms of toxicity or pharmacologic action of these compounds on the kidney may provide insights into the mechanisms of ototoxicity of these agents. This review summarizes the mechanism of action of these drugs on the kidney, and the implications of this action regarding ototoxicity.

Address for communication: John G. Searle Professor and Chair, Department of Internal Medicine, University of Michigan Medical School, 3101 Taubman Center, Ann Arbor, MI 481090368. Phone: 734/936-4495; fax: 734/936-7024; e-mail: dhumes@umich.edu 


\section{AMINOGLYCOSIDE ANTIBIOTICS}

Aminoglycosides have been clearly shown to have both substantial nephrotoxic and ototoxic effects in clinical use. Aminoglycosides are freely filtered by the renal glomerules and transported into the proximal tubule of the kidney. Because the metabolic clearance of aminoglycosides from the tubule cell is slow, this compound accumulates within these cells. If the dose is substantial and prolonged, toxic concentrations accumulate within the cells, ultimately inducing cell injury, cell necrosis, and acute renal failure. ${ }^{1}$ The biochemical interactions of the aminoglycosides and renal tubule cell components have been well documented, with direct effects on phosphoinositide metabolism and mitochondrial bioenergetics. Similar findings regarding phospholipid metabolism have been observed in the inner-ear cells. ${ }^{2}$ More recently, an important role for aminoglycoside-induced free-radical generation as a pathogenetic mechanism for renal tubule cell injury has been demonstrated. ${ }^{3}$ Aminoglycosides promote iron mobilization from mitochondria by hydrogen peroxide. In fact, scavengers of reactive oxygen metabolites as well as iron chelators are able to prevent aminoglycoside nephrotoxicity in animal models. ${ }^{4}$

The pathophysiology of cochlear and vestibular damage by aminoglycosides has been due to the selective destruction of hair cells, beginning in the cochlea with the outer hair cells of the lower turns. ${ }^{5}$ This pathologic event results in high-frequency hearing loss progressing to lower-frequency hearing loss. Unlike the kidney, this ototoxicity is not associated with high levels of aminoglycosides in inner-ear fluids, so selective toxicity cannot be explained by high penetration and accumulation of these antibiotics within inner-ear tissues. ${ }^{6}$ Similar to the kidney, a role for free radical-induced damage to the inner ear promoted by these compounds has been demonstrated. Protection of aminoglycoside-induced hearing loss has been seen with simultaneous administration of iron chelators and free-radical scavengers in animal experiments. ${ }^{7}$ This effect was not associated with a change in serum levels of aminoglycosides, nor with antimicrobial efficacy. These insights may have important implications for the prevention of aminoglycoside toxicity in clinical situations.

\section{CIS-PLATINUM}

cis-Platinum, as with aminoglycoside antibiotics, causes nephrotoxic damage to renal proximal tubules with resulting renal functional impairment. Transport inhibition of cis-platinum into proximal tubules prevents this toxic damage. ${ }^{8}$ This toxic effect also appears to relate to the development of iron-induced free-radical generation, since iron chelators and free-radical scavenging agents prevent renal damage. ${ }^{8}$ In the inner ear, pathologic evaluation demonstrates that cis-platinum promotes degenerative changes in cochlear hair cells. ${ }^{9}$ Hearing loss in treated patients correlates to cumulative dose, suggesting that toxic concentrations in inner-ear fluids or within hair cells promote these changes. ${ }^{10,11}$ No studies have probed into the biochemical mechanisms of cis-platinum ototoxicity, but the similarity observed between other nephrotoxic and ototoxic actions of various compounds suggests that free radicals are likely to be involved. 


\section{LOOP DIURETICS}

The loop diuretics, including furosemide and ethacrynic acid, are direct inhibitors of the $\mathrm{Na}^{+}-\mathrm{K}^{+}-2 \mathrm{Cl}^{-}$transport system found along the tubule cells of the loop of Henle in the kidney. ${ }^{12}$ This transport inhibition results in a substantial decline in chloride transport, with resulting solute and water diuresis. This transporter has been found along the marginal and dark cells within the stria vascularis of the inner ear, which are responsible for endolymph secretion. ${ }^{13}$ Inhibition of this transport system may relate to the ototoxic potential of these compounds due to the resulting changes in the ionic composition and fluid volume within the endolymph. Edema within the stria vascularis and a decrease in endocochlear potential have been described with the administration of these drugs. ${ }^{14.15}$

The toxic or functional mechanisms of aminoglycoside antibiotics, cis-platinum, and loop diuretics on the kidneys have direct analogies to those seen in the inner ear. Because renal tissue and renal tubule cells are more readily accessible, elucidation of the biochemical and molecular mechanisms responsible for nephrotoxicity can provide insight and potential applicability to the understanding of the ototoxicity of these agents.

\section{REFERENCES}

1. Humes, H. D. 1988. Aminoglycoside nephrotoxicity. Kidney Int. 33: 900-911.

2. Sснаснт, J. 1993. Biochemical basis of aminoglycoside ototoxicity. Otolaryngol. Clin. North Am. 26(5): 845-856.

3. Ueda, N., B. Guidet \& S. V. Shah. 1993. Gentamicin-induced mobilization of iron from renal cortical mitochondria. Am. J. Physiol. 265(3, Pt 2): F435-F439.

4. Baliga, R., N. Ueda, P. D. Walker \& S. V. Shah. 1997. Oxidant mechanisms in toxic acute renal failure. Am. J. Kidney Dis. 29(3): 465-477.

5. Sha, S. H. \& J. Schacht. 1997. Prevention of aminoglycoside-induced hearing loss. Keio J. Med. 46(3): 115-119.

6. Henley, C. M., III \& J. Schacht. 1988. Pharmacokinetics of aminoglycoside antibiotics in blood, inner-ear fluids and tissues and their relationship to ototoxicity. Audiology 27(3): 137-146.

7. SCHACHT, J. 1998. Aminoglycoside ototoxicity: prevention in sight? Otolaryngol--Head Neck Surg. 118(5): 674677 .

8. Ban, M., D. Hetrich \& N. Huguet. 1994. Nephrotoxicity mechanism of cis-platinum (II) diamine dichloride in mice. Toxicol. Lett. 71(2): 161-168.

9. Wright, C. G. \& S. D. SChaEfER. 1982. Inner ear histopathology in patients treated with cis-platinum. Laryngoscope 92(12): 1408-1413.

10. Moroso, M. J. \& R. L. Blair. 1983. A review of cis-platinum ototoxicity. J. Otolaryngol. 12(6): 365-369.

11. Marco-Algarra, J., J. Basterra \& J. Marco. 1985. cis-Diaminedichloro platinum ototoxicity. An experimental study. Acta Oto-Laryngol. 99(3-4): 343-347.

12. Wilcox, C. S. 1988. Diuretics and potassium. In The Regulation of Potassium Balance, D. W. Seldin and G. Giebisch, Eds.: 325-345. Raven Press. New York.

13. Ferrary, E. \& O. Sterkers. 1998. Mechanisms of endolymph secretion. Kidney Int. 53(65): S-98-S-103. 
14. RYBAK, L. P. 1993. Ototoxicity of loop diuretics. Otolaryngol. Clinics North Am. 26(5): 829-844.

15. Arnold, W., J. B. Nadol, JR. \& H. Weidauer. 1981. Ultrastructural histopathology in a case of human ototoxicity due to loop diuretics. Acta Oto-Laryngol. 91(5-6): 399414. 